2002). Although parental psychiatric disorder might increase the chance that children become looked after, this is not inevitable and there are many other reasons why children may enter the care system. In fact, only $6 \%$ of the children participating in the survey on which our analysis was based were accommodated primarily as a result of any type of parental illness.

As the survey involved no contact with the biological parents of participants and historical information about children who are looked after is notoriously scarce, we had no way of accurately assessing the mental health of the biological parents. Our paper refers to our frustration at the extremely limited amount of information available to the survey and in clinical practice, and we explicitly state that our analysis cannot be seen as covering all potential risk and resilience factors.

Even if we had access to data on the mental health of the biological parents, an excess of children with psychiatric disorder among parents with psychiatric disorder would not necessarily indicate a biological or genetic basis for this finding. The mean age that children participating in this survey entered the care system was between 7 and 8 years, and we know that mental illness can have an impact on parenting practices. Do the children of parents with mental illness have raised rates of psychiatric difficulties as a result of genetic vulnerability and/or exposure to maladaptive parenting, or perhaps both processes occur at the same time and/or moderate each other? The literature suggests that parenting is an important mediating variable, although other genetic and environmental factors also play a part in the familial aggregation of psychopathology (Ramachandani \& Stein, 2003). Crosssectional surveys are not able to disentangle such complex questions, as data about exposures and outcomes are gathered at the same time. Longitudinal designs would be needed to explore Dr Sekar's theory.

Ford, T., Vastanis, P., Meltzer, H., et al (2007)

Psychiatric disorder among British children looked after by local authorities: comparison with children living in private households. British Journal of Psychiatry, 190. 319-325.

Ramachandani, P. \& Stein, A. (2003) The impact of parental psychiatric disorder on children. BMJ, 327, 242243.

Rutter, M. (2002) Nature, nurture, and development: from evangelism through science to policy and practice. Child Development, 73, I-2I.
Rutter, M., Moffitt, T. E. \& Caspi, A. (2006) Geneenvironment interplay and psychopathology: multiple varieties but real effects. Journal of Child Psychology and Psychiatry, 47, 226-261.

Stein, E., Evans, B., Mazumber, R., et al (1996) The mental health of children in foster care: a comparison with community and clinical samples. Canadian Journal of Psychiatry, 4I, 385-39l.

T. Ford Institute of Psychiatry, King's College London, UK. Email: t.ford@iop.kcl.ac.uk

P. Vostanis Department of Health Sciences, Leicester University, UK

H. Meltzer, R. Goodman Institute of Psychiatry, King's College London, UK

doi: 10.1192/bjp.191.4.360b

\section{Lithium for prevention of Alzheimer's disease}

Nunes et al (2007) reported that the prevalence of Alzheimer's disease in a group of elderly patients with bipolar disorder who were on continuous lithium treatment was significantly less than in a similar group without recent lithium therapy. After controlling for age, lithium use remained associated with a smaller risk of Alzheimer's disease (age-adjusted OR $=0.079,95 \%$ CI 0.020-0.321). Conversely, Dunn et al (2005) showed that patients who received lithium had a significantly higher risk of dementia than those who did not (ageadjusted $\mathrm{OR}=1.8,95 \%$ CI 1.1-2.8).

Nunes et al (2007) found no differences between the lithium and the comparison group in neuropsychological performance after excluding patients with Alzheimer's disease. This is in accordance with our study using Mini-Mental State Examination (MMSE) scores (Terao et al, 2006). Our study, however, showed that patients with present and/or past history of lithium treatment had significantly better MMSE scores than patients without any history of lithium treatment (Terao et al, 2006). It is important to further investigate lithium in the prevention of Alzheimer's dementia with a large number of patients in prospective studies.

If lithium has a preventive effect for Alzheimer's disease, there may be two possible mechanisms. First, it might indirectly prevent dementia via its prophylactic effects on mood disorders, because the rate of dementia increased $13 \%$ with every episode leading to admission for patients with depressive disorder and $6 \%$ for patients with bipolar disorder, when adjusted for differences in age and gender (Kessing $\&$ Andersen, 2004). Second, lithium might directly prevent dementia via its inhibition of glycogen synthase kinase 3 (GSK-3) alpha (Phiel et al, 2003) and GSK-3 beta (Phiel \& Klein, 2001). Although Nunes et al (2007) found no significant differences in the number of previous depressive and manic episodes between the lithium and comparison groups, at present both possibilities should be borne in mind.

Dunn, N., Holmes, C. \& Mullee, M. (2005) Does lithium therapy protect against the onset of dementia? Alzheimer Disease and Associated Disorders, 19, 20-22.

Kessing, L.V. \& Andersen, P. K. (2004) Does the risk of developing dementia increase with the number of episodes in patients with depressive disorder and in patients with bipolar disorder? Journal of Neurology, Neurosurgery and Psychiatry, 75, 1662-1666.

Nunes, P. V., Forlenza, O. V. \& Gattaz, W. F. (2007) Lithium and risk for Alzheimer's disease in elderly patients with bipolar disorder. British Journal of Psychiatry, 190, 359-360.

Phiel, C. J. \& Klein, P. S. (200I) Molecular targets of lithium action. Annual Review of Pharmacology and Toxicology, 4I, 789-8I3.

Phiel, C. J., Wilson, C. A., Lee, V. M.-Y., et al (2003) GSK-3 alpha regulates production of Alzheimer's disease amyloid-beta peptides. Nature, 423, 435-439.

Terao, T., Nakano, H., Inoue, Y., et al (2006)

Lithium and dementia: a preliminary study. Progress in Neuro-Psychopharmacology and Biological Psychiatry, 30, II25-II28.

T. Terao Oita University Faculty of Medicine, Oita, Japan. Email: terao@med.oita-u.ac.jp doi: 10.II92/bjp.19I.4.36I

Authors'reply: Dunn et al (2005) identified from the General Practice Research Database in the UK all cases of dementia between 1992 and $2002(n=9954)$ and compared the number of prescriptions of lithium for individuals with this diagnosis with a control group without dementia $(n=9374)$. They found that more patients with dementia $(n=47,0.47 \%)$ than controls $(n=40,0.43 \%)$ were exposed to lithium. We feel that this finding does not allow conclusions to be drawn as to whether lithium protects against or confers a risk for dementia, because it has been shown that patients with dementia have an increased risk of developing mania and depression (Nilsson et al, 2002) and are thus more likely to receive treatment, including lithium. Conversely, affective disorders themselves seem to increase the risk for dementia.

In a series of studies based on data from the Danish psychiatric case register, Kessing et al found that $14 \%$ of elderly patients with bipolar disorder and $16 \%$ with 